\title{
Matéria
}

ISSN 1517-7076

\section{Análise de um Compósito Complexo por Microscopia Eletrônica Digital e Análise de Imagens}

\author{
Júlia Gomes Ázara de Oliveira, Amelia Angélica Ulloa Torres, Sidnei Paciornik, José Roberto Moraes \\ d’Almeida, Marcos Henrique de Pinho Maurício \\ Pontifícia Universidade Católica do Rio de Janeiro - PUC-Rio - Dept ${ }^{0}$ de Ciência dos Materiais e \\ Metalurgia, Rua Marquês de São Vicente, 225, CEP 22453-900, Rio de Janeiro, RJ, Brasil \\ e-mail: julia_azara@yahoo.com.br, ayelica@yahoo.es, sidnei@dcmm.puc-rio.br, \\ dalmeida@dcmm.puc-rio.br, marcosh@dcmm.puc-rio.br
}

\section{RESUMO}

Um tubo fabricado com material compósito de matriz polimérica reforçado por fibras de vidro foi caracterizado através de microscopia eletrônica digital. Uma seção circunferencial do tubo foi observada em um microscópio eletrônico de varredura com captura digital de imagem. Um mosaico com imagens em alta magnificação foi gerado para que fosse possível uma caracterização que abrangesse desde as fibras individualmente até a distribuição espacial das camadas de fibras. Através do desenvolvimento de uma seqüência de processamento, dados como o diâmetro das fibras e ângulo de enrolamento foram obtidos, bem como foi realizada a identificação das várias camadas de fibras presentes.

Palavras chaves: compósitos, microscopia digital, enrolamento filamentar.

\section{Analysis of a Complex Composite through Digital Electron Microscopy and Image Analysis}

\section{ABSTRACT}

A tube made with a glass-fiber reinforced polymer-matrix composite material was characterized through digital microscopy. A circumferential section of the tube was observed in a Scanning Electron Microscopy with digital image acquisition. A mosaic of high magnification images was created in order to allow complete characterization of the material. Through the development of an image analysis method, the fiber diameters, the winding angles and the discrimination of the layers were obtained.

Keywords: composites, digital microscopy, filament winding, image analysis.

\section{INTRODUÇÃO}

Materiais compósitos de matriz polimérica reforçados por fibras vêm sendo cada vez mais empregados em estruturas de alto desempenho mecânico. Esses materiais apresentam como vantagens em relação aos materiais convencionais, elevadas propriedades específicas - ou seja, altas razões resistência / densidade e módulo de elasticidade / densidade - e excelente resistência à corrosão [1]. Assim sendo, existe atualmente um crescente interesse em empregar dutos fabricados em compósitos em instalações de exploração de petróleo e gás.

O emprego otimizado de dutos compósitos nas mais diversas situações operacionais encontradas no dia a dia em uma instalação industrial requer um projeto preciso do compósito e também requer um controle cuidadoso das variáveis do processo de fabricação, de modo a que a microestrutura final do compósito seja a mais uniforme possível. De fato, o controle da microestrutura e sua posterior caracterização é uma etapa fundamental no desenvolvimento de qualquer material devido a correlação microestrutura / propriedades de um material. Para compósitos reforçados por fibras, em particular, o detalhamento da microestrutura é uma etapa fundamental devido a direcionalidade das propriedades, que variam em função da disposição espacial das fibras.

Neste trabalho é apresentado o desenvolvimento de um método de análise digital de imagens para a caracterização de tubos de resina poliéster reforçada por fibras de vidro. Utilizou-se o método de montagem de mosaicos e foram obtidos dados relativos a geometria das fibras e as orientações das diferentes camadas. 


\section{EXPERIMENTAL}

\subsection{Seleção e Preparação de Amostras}

A amostra utilizada no trabalho foi retirada de um tubo de resina poliéster reforçada por fibras de vidro, com 200 mm de diâmetro e fabricado por Enrolamento Filamentar, sendo este de fabricação nacional e utilizado para transporte de águas de serviço em plataformas de produção de petróleo.

Para a caracterização microestrutural, a amostra foi retirada na direção circunferencial do tubo, indicada na Figura 1.

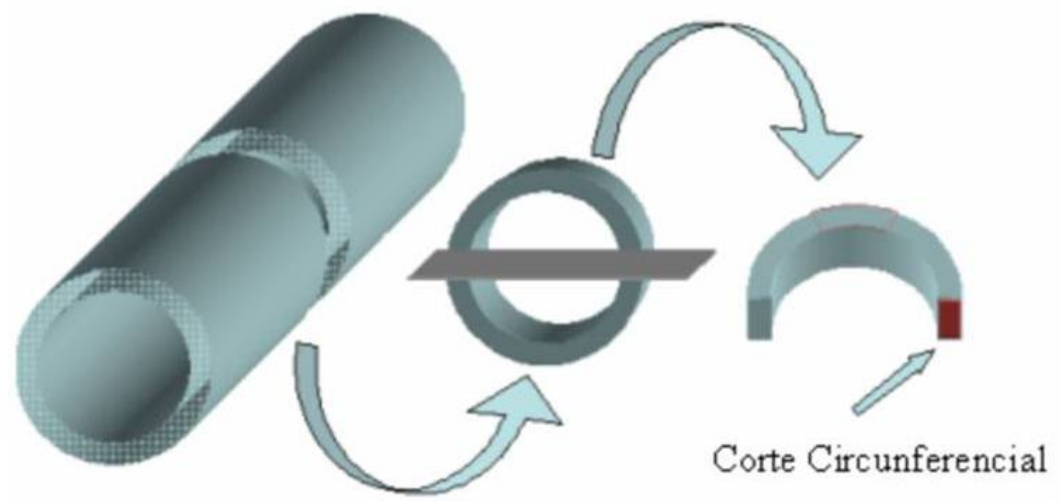

Figura 1: Representação esquemática do corte circunferencial.

A amostra foi então lixada e polida, desde a lixa 80 até pasta de alumina com 0,5 $\mu \mathrm{m}$.

\subsection{Microscopia Digital}

A amostra foi observada no Microscópio Eletrônico de Varredura (MEV), no modo de elétrons retro-espalhados (BSE), sendo as imagens capturadas com resolução de 512 x 480 pixels e aumento de 200X. Como o contraste do modo BSE é determinado pela diferença de pesos atômicos entre os elementos constituintes do material, mesmo havendo uma grande dificuldade na preparação de amostras, as imagens obtidas não revelaram os defeitos oriundos do corte e do polimento, além de apresentarem ótimo contraste entre a matriz e as fibras.

Uma vez que se desejava caracterizar o compósito quanto à distribuição das fibras e das características destas nas respectivas camadas, tornou-se necessária a obtenção de uma imagem que conjugasse alta magnificação com campo suficientemente grande para a análise. Tais exigências não poderiam ser satisfeitas por uma única imagem, surgindo a necessidade de se capturar várias imagens em mesmo aumento e em coordenadas cautelosamente escolhidas para que pudessem ser concatenadas, formando um mosaico. Para a montagem apresentada neste trabalho, foram utilizadas 28 imagens, sendo 7 destas posicionadas na direção y e 4 na direção x

\section{RESULTADOS E DISCUSSÃO}

\subsection{Processamento e Análise das Imagens de Mosaico}

Após a captura, realizou-se a equalização da iluminação das 28 imagens, o que permitiu que as junções entre os campos não se tornassem visíveis após a montagem do mosaico. O resultado pode ser observado na Figura 2. 

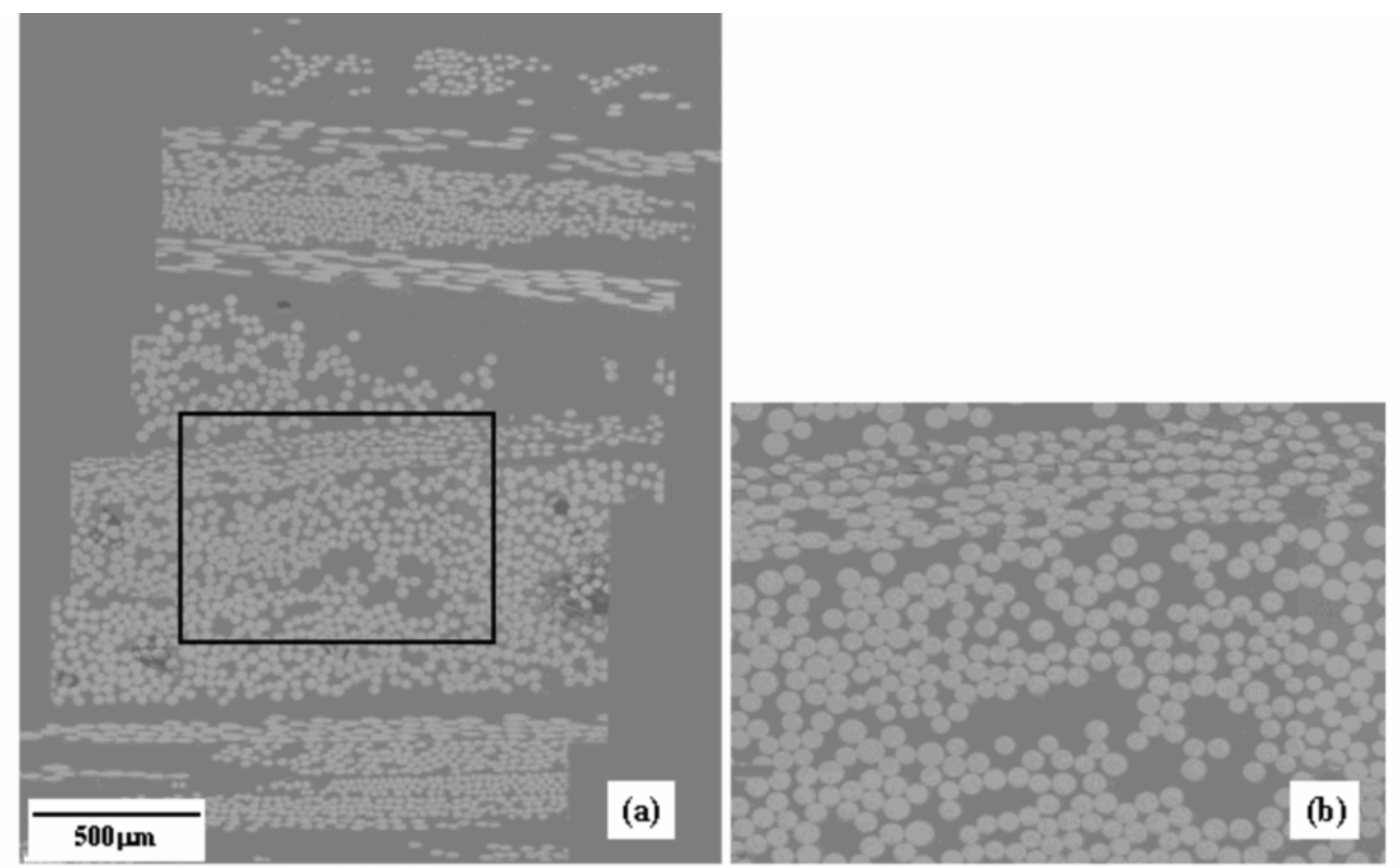

Figura 2: (a)Mosaico 4x7 resultante da junção das 28 imagens obtidas em MEV após equalização da iluminação. (b) Detalhe do mosaico.

Para que fosse possível a caracterização, tornou-se necessária a distinção entre as fibras, alvo da análise, e a matriz. A operação utilizada com tal finalidade denomina-se segmentação [2]]. Ela permite a distinção entre os objetos que devem ser analisados e um fundo que deve ser desconsiderado. Para tal, um tom de cinza intermediário ao tom dos objetos e ao tom do fundo deve ser encontrado; quanto maior o contraste entre os elementos a serem discriminados, maior a facilidade de segmentação. Visto que o modo BSE fornece excelente contraste entre a matriz e as fibras, foi possível utilizar um modo automático [3]], no qual o programa de processamento de imagens determina o tom de corte sem interferência do operador. A partir da segmentação, as fibras passaram a ser os únicos elementos considerados na análise. No entanto, pela proximidade com a qual elas foram depositadas no processo do Enrolamento Filamentar, muitas se tocavam, e após a segmentação tornaram-se um único objeto. Assim, antes de iniciar-se a seqüência para identificação das camadas, tornou-se imprescindível a separação dos objetos que se tocavam. pela utilização do algoritmo do Divisor de Águas [4], cuja eficiência está limitada pelo extensão do contato entre os objetos.

Uma vez obtida a segmentação da imagem e a maior equivalência possível entre o número de fibras e de objetos, iniciou-se o trabalho de separação das várias camadas de enrolamento. Como as fibras apresentavam, na seção do corte, formas elípticas que variavam de acordo com o ângulo de enrolamento quanto mais circular, mais próxima do ângulo de enrolamento $90^{\circ}$ - a forma foi o primeiro parâmetro utilizado para a separação propriamente dita. Com a utilização de parâmetros de forma capazes de avaliar a circularidade de cada objeto []ㅡ, as camadas foram divididas em grupos menores, que por sua vez foram processados de forma independente. Dilatações [6] manualmente controladas tornaram possível a transformação das camadas em grandes objetos, que através de eliminação por tamanho foram isolados um a um. Através de operações lógicas [7] realizou-se o caminho inverso, podendo cada grande objeto voltar à condição de camada constituída por fibras. Em alguns casos, após esta seqüência, novas separações foram realizadas pela aplicação de parâmetros de forma.

No total, foram identificadas 13 camadas de enrolamento na área englobada pelo mosaico 4 x 7 , sendo estas apresentadas na Figura 3. 


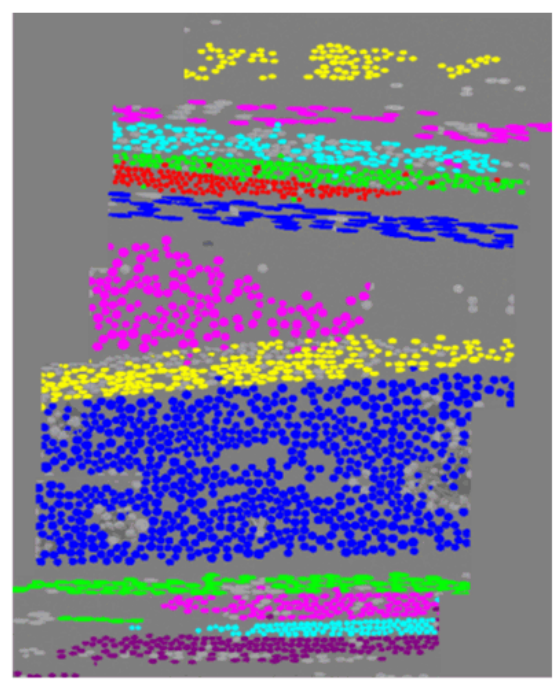

Figura 3: Resultado do processamento.

De cada camada foram automaticamente medidos os diâmetros e ângulos de enrolamento das fibras.

O diâmetro foi obtido pela medição do calibre mínimo de cada fibra, parâmetro equivalente ao menor eixo do objeto, e que portanto, não é influenciado pelo grau de inclinação da fibra.

O ângulo de enrolamento “ $\phi$ ”, por sua vez, foi encontrado pela combinação de dois parâmetros: o calibre mínimo, utilizado para a medição do diâmetro, e o calibre máximo. Este último se refere ao maior eixo do objeto, sendo portanto, diretamente influenciado pelo grau de inclinação da fibra.

$$
\phi=\operatorname{arcsen}\left(\frac{\text { cal.min. }}{\text { cal.max. }}\right) \cdot\left(\frac{180}{\pi}\right)
$$

Percebe-se que quanto mais similares são os valores do calibre máximo e do calibre mínimo, mais próximo de $90^{\circ}$ encontra-se o ângulo de enrolamento. Conforme a diferença entre os parâmetros aumenta, o que equivale a objetos com formas mais elípticas, o ângulo em relação ao eixo do tubo vai diminuindo.

As estatísticas destes parâmetros, por camada, estão apresentadas na Tabela 1.

Tabela 1: Média e desvio padrão dos diâmetros e ângulos de enrolamento das fibras, por camada . As posições das camadas na tabela correspondem às posições na Figura 3.

\begin{tabular}{|c|c|c|c|c|}
\hline Camada & Diâmetro médio $(\boldsymbol{\mu m})$ & Desvio padrão $(\boldsymbol{\mu m})$ & $\phi$ médio (graus) & Desvio padrão (graus) \\
\hline 1 & 12,1 & 1,4 & 38,8 & 4,9 \\
\hline 2 & 20,4 & 11,3 & 14,0 & 3,8 \\
\hline 3 & 12,2 & 2,9 & 34,4 & 11,4 \\
\hline 4 & 12,4 & 1,5 & 49,4 & 5,0 \\
\hline 5 & 12,9 & 1,4 & 63,6 & 5,3 \\
\hline 6 & 24,9 & 20,7 & 10,5 & 3,2 \\
\hline 7 & 19,6 & 3,0 & 63,0 & 4,6 \\
\hline 8 & 12,2 & 3,2 & 36,6 & 10,5 \\
\hline 9 & 19,9 & 2,7 & 63,2 & 4,0 \\
\hline 10 & 38,4 & 17,8 & 7,5 & 4,0 \\
\hline 11 & 12,2 & 3,0 & 36,9 & 10,1 \\
\hline 12 & 13,6 & 1,4 & 64,9 & 2,8 \\
\hline 13 & 12,0 & 2,3 & 41,2 & 8,8 \\
\hline
\end{tabular}


Pela avaliação dos resultados, percebe-se que quanto menor o ângulo de enrolamento da camada, mais imprecisa se torna a análise. Os objetos pertencentes a tais camadas possuíam formas elípticas mais acentuadas que o demais e também foram depositados muito próximos uns dos outros, gerando uma grande superfície de contato entre eles. Diante dessa situação, o algoritmo do Divisor de Águas não foi capaz de realizar a separação dos objetos de forma eficaz. A Análise de Imagens não gerou, dessa forma, resultados confiáveis relativos aos diâmetros das fibras pertencentes a tais camadas, fornecendo, no entanto, valores de ângulos de enrolamento satisfatórios.

\section{CONCLUSÕES}

Visto não haver uma uniformidade na distribuição das fibras ao longo da espessura dos tubos obtidos pelo Enrolamento Filamentar, e a geometria do enrolamento influenciar diretamente nas propriedades mecânicas do material fabricado, a técnica de caracterização apresentada mostra-se como uma poderosa ferramenta no controle do processo de fabricação em questão.

A montagem do mosaico mostrou-se imprescindível, uma vez que caracterizações locais não poderiam descrever a organização global do enrolamento, e conseqüentemente, a descrição detalhada da cada camada e de suas posições relativas, dados essenciais para estabelecer a relação microestrutura/propriedades mecânicas.

A Análise de Imagens permitiu que a caracterização de um compósito complexo, inviável de ser feita manualmente, fosse realizada com eficiência e precisão. Mesmo a rotina do processamento sendo um tanto quanto extensa e não podendo ser utilizada exatamente como gerada para análise de compósitos com outra configuração de fibras, ela representa um grande avanço na área de caracterização de materiais compósitos e constitui uma importante referência para análises posteriores.

\section{BIBLIOGRAFIA}

[1] GIBSON, R.F., Principles of Composite Materials Mechanics, New York, McGraw-Hill, 1994.

[2] RUSS, J.C., Computer Assisted Microscopy, The Measurement and Analysis of Images. New York, Plenum Press, 1990.

[3] OTSU, N.A, “Threshold Selection Method from Grayscale Histograms”, IEEE Trans. Sys. Man. Cyb. SMC-9, pp. 62-66, 1979.

[4] BEUCHER, S., LANTEJOUL, C., “Use of Watersheds in Contour Detection”, In: Proc. Int. Workshop Image Processing, Real-time Edge and Motion Detection/Estimation, CCETT/INSA/IRISA, IRISA Report n. 132, Rennes, France, pp. 2.1-2.12, 1979.

[5] PACIORNIK, S., MAURICIO, M.H.P., "Digital Imaging”. In: VANDER VOORT, G. (ed), ASM Handbook, ed. 1, v. 9, pp. 368-402, Ohio, USA, Materials Park, 2004.

[6] SERRA, J., Image Analysis and Mathematical Morphology, Academic Press, 1982.

[7]GONZALEZ, R.C., WOODS, R.E., Digital Image Processing, New York, Addison-Wesley, 1993. 